\title{
Students' Conception on Multiple Integrals of a Function of Several Variables: A Case of Adama Science and Technology University
}

\author{
Eyasu Gemechu ${ }^{1, ~ *}$, Amanuel Mogiso ${ }^{1}$, Yusuf Hussein ${ }^{1}$, Gedefa Adugna ${ }^{2}$ \\ ${ }^{1}$ Department of Mathematics, Faculty of Natural Science and Computation, Wolkite University, Wolkite, Ethiopia \\ ${ }^{2}$ Department of Mathematics, Faculty of Natural Science and Computation, Adama Science and Technology University, Adama, Ethiopia
}

Email address:

gmeyasu@gmail.com (E. Gemechu)

${ }^{*}$ Corresponding author

To cite this article:

Eyasu Gemechu, Amanuel Mogiso, Yusuf Hussein, Gedefa Adugna. Students' Conception on Multiple Integrals of a Function of Several Variables: A Case of Adama Science and Technology University. American Journal of Applied Mathematics. Vol. 9, No. 1, 2021 , pp. 10-15. doi: 10.11648/j.ajam.20210901.12

Received: February 1, 2021; Accepted: March 11, 2021; Published: March 17, 2021

\begin{abstract}
The study was conducted at Adama Science and Technology University to investigate students' conceptual understanding in learning Applied Mathematics II in general and multiple integrals in particular. A case study research design was employed on a Mechanical engineering group one student. This group was randomly selected through simple random sampling techniques. The number of students involved in this study was 50. Qualitative data were collected through reasoning part of the multiple choice items of the pre-test and interview items of the post-test were analyzed using APOS analysis based on proposed genetic decompositions. These tools were intended to investigate the conceptual understanding of students and the way they justify their answers. The study shows that the majority of the students' conception of multiple integrals could be categorized under action level whereas a few students were categorized under process conception. Students' conceptual understanding on multiple integrals of a function of two variables is a straight forward as that of a function of a single variable, which reveals that students have not developed a new schema for a function of two variables, as different from a function of a single variable. The majority of the respondents was poor at extending previous concepts to the new concept and had difficulty to represent multiple integrals using graph. Thus; the researchers recommended the utilization of an appropriate instructional approach in order to scaffold students' conceptual understanding of multiple integrals.
\end{abstract}

Keywords: APOS Theory, Conceptual Understanding, Functions of Two Variables, Multiple Integrals

\section{Introduction}

Today, Ethiopia is sensing the gradual effect of globalization on economic growth and development. The upsurge of new worldwide competition in science is forcing Ethiopia to examine whether its education system can meet the necessities of the $21^{\text {st }}$ century [1]. Thus, in higher education more attention is given to Science, Technology, Engineering and Mathematics (STEM) across the globe. Ethiopian Ministry of Education is also considered science and mathematics education as a center of attention within academic communities at all levels of education, including higher education [2].

Mathematics is one of the most important courses given to engineering and technology students. Out of mathematics courses offered to students in higher education, Applied Mathematics is one. It is a widely applied discipline in areas of studies like for instance, in science, computer science, and engineering and technology [3]. Especially, Applied Mathematics II is a course offered to undergraduate engineering and technology students as a prerequisite course to the other advanced courses. According to Majid, more than $75 \%$ of engineering courses offered to students are built on mathematical concepts [4]. This indicates that it is impossible to talk about science, engineering and technology without mathematics [5]. Mathematics is considered as a backbone for engineering [6]. It is also considered as a subject who seeks to understand the patterns that infuse both mind and world [7]. 


\section{Statement of the Problem}

In comparison with Applied Mathematics I, there are a few numbers of researches that were conducted on students' conceptual understanding of a function of several variables, particularly on multiple integrals $[8,9]$. And also, there is a little work done on what reflections and mental constructions students need to make in order to understand and solve problems of functions of several variables. So, there is a research gap on students' conceptual understanding of some concepts of a function of several variables like multiple integrals of functions of several variables.

\section{General Objective}

The general objective of the study is to investigate levels of students' conception on multiple integrals. The following are the specific objectives of the study:

1. To determine how students conceptualized concepts of multiple integrals of a function of two variables.

2. To explore the way students represent concepts of multiple integrals using symbolic, algebraic and graphical representations.

\section{Research Questions}

Base on the statement of the problem the following research questions were addressed through the study:

1. How students conceptual on concepts of multiple integrals of a function of two variables?

2. How students represent concepts of multiple integrals using symbolic, algebraic and graphical representations?

\section{Material and Methods}

This study was conducted to investigate levels of students' conception on multiple integrals of a function of two variables. So, qualitative research approach was employed since qualitative research gives more emphasis on understanding the phenomenon under investigation through bringing a word or picture data for thick description and interpretation [10]. In this study, Mechanical engineering group one students were selected through simple random sampling techniques. The number of students involved in this study was 50 .

Two tiered conceptual tests were designed by the researchers. The objectives of the instruments were to investigate conceptual understanding of students and the way they justify their answers. So, eight different questions were prepared beforehand, and each question has four alternatives and a reasoning part why they were chosen. The face and content validity of the instruments were ensured through a panel of experts working on the area. Based on the comments of those experts, necessary improvements were made and the revised tool was piloted before the actual administration to the students. Based on the result of the pilot study, the initial genetic decomposition proposed for the concepts of functions of several variables. Four different activities were set to help students make constructions predicted. Those are:

1) Students were asked to define, find and integrate functions of a single variable.

2) Students were also asked to extend definitions, properties and procedures to evaluate integrals in a function of a single variable to a function of two variables.

3) Students were also asked to convert word problems given to the students to algebraically, or symbolically representation.

4) Students were also asked to graphically represent any algebraically represented multiple integrals a function of two variables.

The qualitative data collected were analyzed through thematizing students' reasoning and interview result into four different areas like students' conception related to definition, extending definition to a new concept, algebraic representation and graphical representation. Thus, students' responses were categorized into different mental constructions as per APOS theory based on genetic decomposition proposed. This enables the researcher to know the students' level of conception.

\section{Results and Discussion}

One of the conceptions of a definite integral $\int_{a}^{b} f(x) d x$ is an area of the plane region bounded by the curve $y=f(x)$, the $x$-axis and the lines $x=a$ and $x=b[11,12]$. None of the respondents have difficulty in defining, algebraically or symbolically, and graphically represent function of a single variable. They do have know-how of the concept to integrate function of a single variable. They know when to utilize different properties and rules of integration.

Similarly, students were asked to extend integration of a function of a single variable to double integral of $f(x, y)$ over the domain D i.e. $\iint_{D} f(x, y) d A$, in such a way that its value will give the volume of the solid $\mathrm{S}$ whenever $\mathrm{D}$ is a domain and $f$ is its function with positive values. So, students were probed to determine limits of integration and reason out why for $\int_{0}^{1} \int_{\sqrt{x}}^{1} e^{y^{3}} d y d x .15$ students out of 50 reasoned out that it is impossible to solve the given integration as it is. They replied that it needed to be reversed. About 23 of the respondents replied it is possible to solve the given integration as it is. About 12 of the respondents' reasons show that it is possible to reverse the order of integration simply by interchanging the place of $d x$ and $d y$ or simply by applying Fubini's theorem as it is. This reveals that the majority of students' conception on reversing the order of integration is at an active level as proposed in genetic decomposition.

On top of this, students were asked to reason out the concept of Fubini's theorem. 24 out of 50 students clearly indicates that the theorem works if $f(x, y)$ is a continuous function, then $\int_{a}^{b} \int_{c}^{d} f(x, y) d y d x=\int_{c}^{d} \int_{a}^{b} f(x, y) d x d y .12$ of the respondents gave their reasoning as if $f(x, y)$ is a 
continuous function, then $\int_{a}^{b} \int_{c}^{d} f(x, y) d y d x=$ $\int_{c}^{d} \int_{a}^{b} f(x, y) d y d x .8$ respondents justify it as if $f(x, y)$ is a continuous function, then $\int_{a}^{b} \int_{c}^{d} f(x, y) d y d x=$ $\int_{c}^{d} f(x, y) d x \cdot \int_{a}^{b} f(x, y) d y$. The rest of the respondents were replied as if $f(x, y)$ was continuous over the region $\mathrm{D}$, then

$\iint f(x, y) d x d y,=\iint f(r, \theta) \theta d r d \theta$ when we see the studerfts' understanding of the Fubini's theorem, the majority of the respondents answered correctly. This reveals that the majority of students' conception can be categorized under process level as proposed in genetic decomposition.

Students were also asked to algebraically represent horizontal simple regions of a given double integral i.e. $\iint_{R} f(x, y) d A, 13$ respondents out of 50 replied as $R=$ $\left\{(x, y): a \leq y \leq b, g_{1}(y) \leq x \leq g_{2}(y)\right\}$. About 6 of the respondents represent it as $R=\left\{(x, y): a \leq x \leq b, g_{1}(x) \leq\right.$ $\left.y \leq g_{2}(x)\right\}$. About 9 of the respondents represent it as $R=\left\{(x, y): a \leq x \leq b, g_{1}(x) \leq x \leq g_{2}(x)\right\}$ whereas the remaining respondents represent it as $R=\{(x, y): a \leq y \leq$ $\left.b, g_{1}(x) \leq y \leq g_{2}(x)\right\}$. This implies that the majority of the respondents had difficulty in algebraic representation of regions of integration. This can be due to the lack of understanding of the students. The easiest way to master regions of integration is the usage of graphical method. So, this indicates that a student's graph conception is poor, which in turn shows that students' process conception is weak.

Moreover, students were probed by $\iint_{R} e^{-\left(x^{2}+y^{2}\right)} d A$, where $R$ is the disc of radius $a>0$ centered at the origin to determine the limit of the integral. About 19 out of 50 replied that $0 \leq \theta \leq 2 \pi$ and $0 \leq r \leq a$ whereas about 9 and 12 respondents reason it out as $0 \leq \theta \leq \pi$ and $0 \leq \rho \leq a$ and $0 \leq \theta \leq$ aand $0 \leq r \leq a$ respectively. The majority of the students correctly reasoned out and converted it to a cylindrical coordinate system. This implies that students understand the process of converting one coordinate system to the other simple region. Thus, they achieved a process conception as per proposed genetic decomposition.

Students were asked to give their reasons for sketching integrations, computing integrals and determining volumes. Thus, students were given height, length and width of a parallelepiped to be $4 \mathrm{~cm}, 3 \mathrm{~cm}$ and $3 \mathrm{~cm}$ respectively, and they were asked to determine the algebraic representation where one of the vertices of the parallelepiped lies at the origin and its base is a square. Only 17 students correctly answered i.e., $\mathrm{V}=\int_{0}^{3} \int_{0}^{3} 4 d y d x$. About 16 of the respondents replied that $\mathrm{V}=\int_{0}^{4} \int_{0}^{3} 3 d y d x, 12$ of them replied as $\mathrm{V}=$ $\int_{0}^{4} \int_{0}^{3} d y d x$ and 5 of them replied as $\mathrm{V}=\int_{0}^{3} \int_{0}^{3} 3 d y d x$ Few students tried to sketch the graph on 3D.

If $f(x, y) \geq 0$ on $\mathrm{D}$, then students were required to determine the place where the volume $(\mathrm{V})$ between the function and the region $\mathrm{D}$ exists. 18 respondents replied that $\iint_{D} f(x, y) d A=V \geq 0$, where $\mathrm{V}$ is the volume of the solid lying vertically above $\mathrm{D}$ and below the surface $z=f(x, y)$. About 14 respondents replied that $\iint_{D} f(x, y) d A=V \geq 0$, where $\mathrm{V}$ is the volume of the solid lying vertically below D and above the surface $z=f(x, y) .9$ of the respondents reasoned out as $\iint_{D} f(x, y) d A=V \geq 0$, where $\mathrm{V}$ is the volume of the solid lying vertically above $\mathrm{D}$ and below the surface $z=f(x, y)$ and 9 of them replies as $\iint_{D} f(x, y) d A=-V \leq 0$, where $\mathrm{V}$ is the volume of the solid lying vertically below D and above the surface $z=f(x, y)$.

If $f(x, y) \leq 0$ on $\mathrm{D}$, then students were required to determine the place where the volume (V) between the function and the region D exists. Majority of the students (19) replied that $\iint_{D} f(x, y) d A=-V \leq 0$, where $\mathrm{V}$ is the volume of the solid lying vertically below D and above the surface $z=f(x, y) \quad 13$ students replied that $\iint_{D} f(x, y) d A=V \geq 0$, where $\mathrm{V}$ is the volume of the solid lying vertically below $\mathrm{D}$ and above the surface $z=f(x, y) 11$ students replied that $\iint_{D} f(x, y) d A=-V \leq 0$, where $\mathrm{V}$ is the volume of the solid lying vertically above D and below the surface $z=f(x, y)$ and 7 students replied that $\iint_{D} f(x, y) d A=V \geq 0$, where $\mathrm{V}$ is the volume of the solid lying vertically above $\mathrm{D}$ and below the surface $z=f(x, y)$.

In addition to this, students were given a function $f(x, y)$ that is defined on a rectangular region as shown in the figure below on $\mathbb{R}$ where $\mathbb{R}=\{(x, y): a \leq x \leq b, c \leq y \leq d\}$. They were asked to determine the double integral in the region below $f(x, y)$ and above the $x y$-plane. 19 students replied correctly as the double integral of $f$ is the region below $z$ and above $x y$-plane. About 13 students replied it as the double integral of $f$ is the region above $z$ and abovethexy-plane. About 10 of the students replied it as the double integral of $f$ is the region below $z$ and below $x y$ plane and 8 of the students replied it as the double integral of $f$ is the region above $z$ and below $x y$-plane.



Figure 1. Graphical representation of multiple integral. 
In line with this, those who were found to have a better reasoning were those who were taught with the support of MATLAB. This was so because MATLAB assisted them to physically visualize the region and the overall sketch of the integration. Despite these groups, many others had difficulties with their work and reasons listed below.

1) They had difficulties with finding the limits of multiple integrals

2) They had difficulty in reverse order of integration from horizontal region to vertical region and vice versa.

3) Lack of understanding of double integrals in functions of several variables as the volume of the solid region bounded by the surface $z=f(x, y)$ and the $x y$-plane.

4) They had difficulty in sketching a graph and understanding the graph of solid regions, etc.

The researcher interviewed some selected students to investigate students' conceptual understanding of some concepts of double integral like on iterated integrals, reversing the order of integration, determining the limit of integration, sketching graphs of the region of integration and so forth. Some results of the interview were discussed as follows:

Among the students interviewed on concepts of multiple integrals of functions of several variables, all of them showed an action conception. They can be able to carry out the iterated integral of the rectangular region. They can able to determine the limits of integration for iterated integrals of functions based on properties of multiple integrals. In contrary to this, they had difficulties in reversing the order of integration in the case of general regions.

There are some students demonstrating a lack of understanding of a function of several variables. These students demonstrated difficulties associated with different actions, such as if techniques of integration (integration by substitution, integration by part, etc.). This difficulty fixed them to action level. On top of this, it is observed that there is a tendency of memorized facts in their responses. For instance, S11 responded to multiple integrals of functions of several variables $\int_{0}^{1} \int_{0}^{x}\left(x^{2}+y^{2}\right) d y d x$ as "integrating the inner with respect to $d x$ first i.e. $\int_{0}^{x}\left(x^{2}+y^{2}\right) d x$ by considering variable $y$ as constant which could be considered as wrong conception. Besides, the student was probed by $\int_{0}^{1} \int_{2 y}^{2} \cos \left(x^{2}\right) d x d y$ whether it could be solved as it is or not. Then, the respondent tried to solve it using a substitution method by letting $u=x^{2}$ which could be taken as misconception. The respondent could not be able to shift the order of integration. In general, as indicated above, S11 demonstrated difficulty of differentiate which variable is the inner and which is not. Here, the respondent tried to integrate the given function with respect to $d x$. The interviewer insisted that he correct. Later, he did all procedures correctly. In the second case the student tried to apply the same method to evaluate the given integral. This implies that the student is memorizing the former procedure to apply it the new situation. This shows that students had a tendency to memorize facts in their responses which led to action conceptions.
In contrast to this, $\mathrm{S} 15$ could be able to integrate the inner function and then do the outer integration for an iterated integral whereas still challenged to solve $\int_{0}^{1} \int_{2 y}^{2} \cos \left(x^{2}\right) d x d y$. He tried to rewrite the double integral as $\int_{0}^{1} \int_{2 y}^{2} x^{2} \cos d y d x$ and considered it if the order of integration is shifted from $d x d y$ is changed to $d y d x$. Or, we can use $D_{1}<y<D_{2}$. This indicates that he has difficulty in reversing the order of integration from one region to the other. The respondent tried to apply the same method as that of iterated integral to evaluate the new integral. This implies that the student is memorizing the former procedure to apply it to the new situation. This shows that student had a tendency to memorized facts in their responses which led to action conceptions.

Out of the students interviewed, about three of them showed a process conception on multiple integrals of functions of several variables. Their response showed that they have interiorized the actions described in the genetic decomposition throughout questions given to them. At these level students should clearly identify the limit of integration and why they considered different techniques of integration. This is a basic difference between action conception and process conception. If so, they demonstrated that they had interiorized the actions of finding integrals of a given function of several variables. Hence, they can be categorized under process conception as it is proposed in genetic decomposition. For instance, S12 knew which variables should be integrated first when compared with one of his friends above in action level. In the first case he did very well He clearly put all procedures clearly. In the second problem given to him, he understood that the former way of evaluation did not work for the current problem in hand. This implies that he understood that both problems did not follow the same procedures to be solved. But, it was observed that he had difficulty to whether the given integration is integrable as it is or not. So, the interviewer guided him to shift one region to the other, but he was able to do that.

On top of this, students were also asked to graphically represent the given limit of integration. This construction allows the students to work with different regions (horizontal region and vertical region, rectangular coordinate, polar coordinate, cylindrical coordinate and spherical coordinate) of functions of several variables. If they can coordinate to identify the limit of integration of the function within a given domain of the function and use it to determine regions using graph representation, then they can be categorized under the object conception as it is indicated in the proposed genetic decomposition. Among the interviewees, only one student achieved this level. The respondent reversed the order of integration. The interviewee understood that it is a must to reverse the order of integration because it is difficult to solve the double integral as it is. The given region is a horizontal region. So, it is a must to shift the order of integration into the vertical region. He used graph representation for the given region and tried to solve it. In fact, in the middle, he was a little bit confused.

None of the students interviewed demonstrated all 
schemata levels (i.e. action, process, object, and schema) correctly and sufficiently as per proposed genetic decomposition. The majority of the interviewees' mental construction lies under action conception, some of the respondents were categorized under process conception and none of the interviewees were categorized under object level with some lack of conversion. This indicates that there are no interviewees demonstrated a schema conception.

According to Martinez-Planell and Gaisman students better understand functions of two variables in multivariate calculus when they are exposed to graphical representations of the function [9]. Similarly, they conclude that students' understanding is better if they can represent and give concepts using a graph representation [13, 14].

In the attempt to investigate students understanding some probing questions that require reversing the order of integration, few students replied that it must be reversed the order of integration since it is impossible to integrate the inner function as it is. A majority of them, however, replied that it is possible to integrate as it is. Some of them tried to justify the use of Fubini's theorem. These imply that students had difficulty on how to reverse the order of integration and apply it. This seems a direct consequence of their knowledge of direct integration, which caused them to fail realize the issue of reversing orders.

The data reveal that the majority of the students' construction of multiple integrals were categorized under action level where as few students' conception were categorized under process conception. None of the students were categorized under object and schema conception. This indicates that either the students' mathematics background is poor or due to poor instructional approach that teachers were using in the classroom to teach multiple integrals [3]. According to Martinez-Planell and Gaisman students better understand functions of two variables in multivariate calculus when they are exposed to graphical representations of the function [9]. Similarly, they conclude that students' understanding is better if they are able to represent the given concepts using a graph representation [3, 13, 14].

Literature indicates that students' difficulty in understanding multiple integrals goes back to students' understanding of limits and derivatives [15- 17]. Thus, the study shows that students encountered an epistemological problems emanated from the language and symbolism used.

\section{Conclusion}

The study clearly shows that students have difficulty in understanding multiple integrals. On top of this, it was observed that students have epistemological problems emanated from the language and symbolism used. For instance, errors that students made during reversing the order of integration were reported as follows:

1) Some learners did not understand the process of reversing the order.

2) Some could not sketch the regions of integration correctly.
3) A small number of learners could not understand keeping constant and integrating $\mathrm{x}$.

The study shows that students have representations difficulties of concepts of multiple integrals. Thus, this study shows that students were demonstrating difficulties in using appropriate representation for different functions and transfer between the representations with relative ease [18-21].

The data shows students had difficulty in defining multiple integrals of a function of two variables, extending concept to a function of several variables, and symbolically representing multiple integrals of a function of two variables [22-24]. Thus, the study reveals that students were suffering from difficulties like defining, extending definitions to new concepts and representing a given mathematical questions using multiple ways of representation. This implies that students have a lack of conceptual understanding. So, it is better to scaffold students' conceptual understanding using another instructional approach.

\section{References}

[1] Teshome Yizengaw (2004). The Status and Challenges of Ethiopian Higher Education System and its Contribution to Development. The Ethiopian Journal of Higher Education, 1 (1).

[2] MoE (2008). General Education Quality Improvement Package (GEQIP).

[3] Eyasu G., Kassa M. \& Mulugeta A. (2018). MATLAB Supported Learning and Students' Conceptual Understanding of Functions of Two Variables: Experiences from Wolkite University. Bulgarian Journal of Science and Education Policy (BJSEP), 12, (2).

[4] Majid, M. A. (2014). Integrated Technologies Instructional Method to Enhance Bilingual Undergraduate Engineering Students' Achievements in the First Year Mathematics: $A$ thesis submitted for the degree of Doctor of Philosophy, Brunel University, London, United Kingdom.

[5] Winkelman, P. (2009). Perceptions of mathematics in engineering. European Journal of Engineering Education, 34 (4), 305-316.

[6] James, W. \& High, K. (2008). Freshman-Level Mathematics in Engineering: A Review of the Literature in Engineering Education. American Society for Engineering Education.

[7] Schoenfeld, A. H. (1992). Learning to think mathematically: Problem solving, metacognition, and sense-making in mathematics. In D. Grouws (Ed.), Handbook for Research on Mathematics Teaching and Learning (pp. 334-370). New York: MacMillan.

[8] Kerrigan, S. (2015). Student Understanding and Generalization of Functions from Single to Multivariable Calculus. Honors Baccalaureate of Science in Mathematics project submitted to Oregon State University.

[9] Martínez-Planell, R., \& Gaisman, M. T. (2012). Students' understanding of the general notion of a function of two variables. Educational Studies in Mathematics, 81 (3), 365384. 
[10] Tewksbury, R. (2009). Qualitative versus quantitative methods: understanding why qualitative methods are superior for criminology and criminal justice. Journal of theoretical and philosophical criminology, 1 (1).

[11] Adams, R. (2003). Calculus: A Complete Course. Pearson Education Canada Inc., Toronto, Ontario.

[12] Stewart, J. (2008). Calculus: Early Transcendental, $\left(6^{\text {th }}\right.$ ed $)$. Thompson Brooks/Cole.

[13] Trigueros, M., \& Martinez-Planell, R. (2010). Geometrical representations in the learning of two variable functions. Educational Studies in Mathematics, 73 (1), 3-19. doi: 10.1007/s10649-009-9201-5.

[14] Martínez-Planell, R. \&Gaisman, M. T. (2013). Graphs of functions of two variables: Results from the design of instruction, International. Journal of Mathematical Education in Science and Technology, 44 (5), 663-672, DOI: 10.1080/0020739X.2013.780214.

[15] Robert, A. \& Speer, N. (2001). Research in the teaching and learning of calculus /elementary analysis (pp. 283-299). In: Holton, D., Artigue, M., Kirchgräber, Hillel, J., Niss, M. \& Schoenfeld, A. (Eds.). The teaching and learning of mathematics at University level. Berlin: Springer.

[16] Cornu, B. (1981). Advanced Mathematical Thinking. In Nesher, P. and Kilpatrick, J. (eds), Mathematics and Cognition. (pp 113-134).

[17] Orton, A. (1983). Students' Understanding of Integration. Educational Studies in Mathematics, 14, 1-18.
[18] Dubinsky, E., \& Harel, G. (1992). The nature of the process conceptions of function. In G. Harel E. Dubinsky (Eds.), The concept of function: Aspects of epistemology and pedagogy, MAA Notes 25, pp. 85-106). Washington, DC: Mathematical Association of America.

[19] Eisenberg, T., \& Dreyfus, T. (1994). On understanding how students learn to visualize function and transformations. In E. Dubinsky, A. Schoenfeld, \& J. Kaput (Eds.), Research incollegiate mathematics I, 4 (45-68). Providence, RI: American Mathematical Society.

[20] Metcalf, R. C. (2007). The nature of students' understanding of quadratic functions. (PhD Dissertation, State University of New York at Buffalo, Buffalo, NY).

[21] Rockswold, G. K. (2010). College algebra with modeling and visualization $\left(4^{\text {th }}\right.$ ed.). New York: Addison-Wesley.

[22] Akkus, R., Hand, B., \& Seymour, J. (2008). Understanding students' understanding of functions. Mathematics Teaching Incorporating Micromath, 207, 10-13.

[23] Carlson, M., Oehrtman, M., \& Engelke, N. (2010). The precalculus concept assessment: a tool for assessing students' reasoning abilities and understandings. Cognition and Instruction, 28, 113-145. DOI: 10.1080/07370001003676587.

[24] Davis, J. D. (2007). Real world contexts, multiple representations, student-invented terminology, and y-intercept. Mathematical Thinking and Learning, 9, 387-418. DOI: 10.1080/10986060701533839. 